\title{
An RNA ligase-mediated method for the efficient creation of large, synthetic RNAs
}

\author{
MARTHA R. STARK, ${ }^{1,4}$ JEFFREY A. PLEISS, ${ }^{2,4}$ MICHAEL DERAS, ${ }^{3}$ \\ STEPHEN A. SCARINGE, ${ }^{3}$ and STEPHEN D. RADER ${ }^{1}$ \\ ${ }^{1}$ Department of Chemistry, University of Northern British Columbia, Prince George, BC V2N 4Z9, Canada \\ ${ }^{2}$ Department of Biochemistry, University of California, San Francisco, San Francisco, California 94143, USA \\ ${ }^{3}$ Dharmacon RNA Technologies, Lafayette, Colorado 80026, USA
}

\begin{abstract}
RNA ligation has been a powerful tool for incorporation of cross-linkers and nonnatural nucleotides into internal positions of RNA molecules. The most widely used method for template-directed RNA ligation uses DNA ligase and a DNA splint. While this method has been used successfully for many years, it suffers from a number of drawbacks, principally, slow and inefficient product formation and slow product release, resulting in a requirement for large quantities of enzyme. We describe an alternative technique catalyzed by T4 RNA ligase instead of DNA ligase. Using a splint design that allows the ligation junction to mimic the natural substrate of RNA ligase, we demonstrate several ligation reactions that appear to go nearly to completion. Furthermore, the reactions generally go to completion within $30 \mathrm{~min}$. We present data evaluating the relative importance of various parameters in this reaction. Finally, we show the utility of this method by generating a 128-nucleotide pre-mRNA from three synthetic oligoribonucleotides. The ability to ligate synthetic or in vitro transcribed RNA with high efficiency has the potential to open up areas of RNA biology to new functional and biophysical investigation. In particular, we anticipate that sitespecific incorporation of fluorescent dyes into large RNA molecules will yield a wealth of new information on RNA structure and function.
\end{abstract}

Keywords: T4 RNA ligase; ligation; RNA; YOL047c; splint

\section{INTRODUCTION}

Site-specific incorporation of modifications into RNA molecules has been a powerful tool for both biochemical and biophysical investigations of RNA function (Moore and Sharp 1992; Wyatt et al. 1992; Heilek et al. 1995; Teigelkamp et al. 1995; Golden et al. 1996; Chavatte et al. 2001). Improved methods for the chemical synthesis of RNA have greatly facilitated such experiments by allowing a wide variety of nonnatural nucleotides to be placed at almost any position in the RNA (McGregor et al. 1996). The primary limitation of this method is that chemical synthesis becomes prohibitively inefficient beyond $\sim 50$ nucleotides (nt). Consequently, to generate larger RNAs with site-specific modifications it is necessary to join the

\footnotetext{
${ }^{4}$ These authors contributed equally to this work.

Reprint requests to: Stephen D. Rader, Department of Chemistry, University of Northern British Columbia, 3333 University Way, Prince George, BC V2N 4Z9, Canada; e-mail: rader@unbc.ca; fax: (250) 960-5170.

Article published online ahead of print. Article and publication date are at http://www.rnajournal.org/cgi/doi/10.1261/rna.93506.
}

synthetic oligoribonucleotide either to other oligonucleotides or to longer, in vitro transcribed RNA.

The most widely used method for ligating RNA molecules employs a DNA splint to hold the RNA ends in apposition while DNA ligase catalyzes chemical bond formation (Moore and Sharp 1992). The design of DNA ligase-mediated ligation (henceforth referred to as "Dligation") is shown in Figure 1A. DNA ligase recognizes the nicked, double-stranded substrate and catalyzes formation of a phosphodiester linkage between the $5^{\prime}$ phosphate of one oligo (generally referred to as the "donor" oligo) and the free 3' hydroxyl of a second oligo (generally referred to as the "acceptor" oligo). Although this method has been used successfully in many experiments, it frequently produces a low yield of product, takes several hours to reach completion, and requires large amounts of DNA ligase (Moore and Query 2000). These limitations have impeded certain applications of the technique, notably those that require multiple ligation steps.

An alternative method for RNA ligation uses T4 RNA ligase. Like DNA ligase, this enzyme also requires a free $5^{\prime}$-phosphate on the donor substrate, a 3' hydroxyl on the 
A

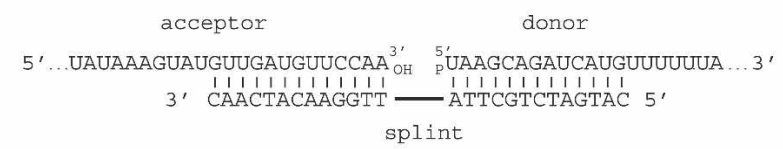

B

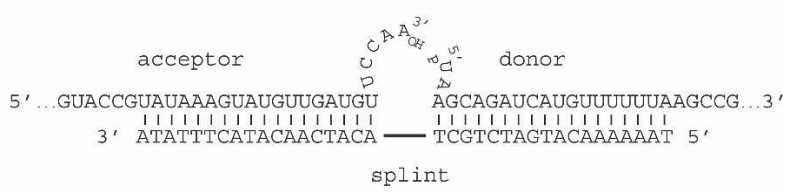

FIGURE 1. Templating schemes for (A) DNA ligase-mediated RNA ligation, and $(B)$ RNA ligase-mediated RNA ligation (sequences shown are YOL047c, junction I). The solid line connecting the two halves of the splint indicates that they are part of a single oligonucleotide. Ligation occurs between the phosphate at the $5^{\prime}$ end of the donor (indicated by "P") and the 3' $\mathrm{OH}$ on the acceptor.

acceptor substrate, and exogenous ATP. However, unlike DNA ligase, RNA ligase is only active when the nucleotides at the splice junction are single stranded. Consequently, RNA ligase works effectively in a nontemplated fashion, indiscriminately joining any nucleotides with the correct $5^{\prime}$ and $3^{\prime}$ termini, a characteristic that has made it useful for techniques such as pCp labeling (England et al. 1980).

In an effort to impose sequence specificity on the reaction, a method was developed based on the observation that the natural substrate of T4 RNA ligase is the anticodon loop of tRNA ${ }^{\text {Lys }}$ (Amitsur et al. 1987). A DNA splint was designed to hold the donor and acceptor molecules near one another while leaving single-stranded regions ("linkers") near the ligation junction (Bain and Switzer 1992; see Fig. 1B). Synthetic yields remained low, however, presumably due to the high efficiency of T4 ligase-mediated circularization (Gumport and Uhlenbeck 1981; Romaniuk and Uhlenbeck 1983), as well as non-optimal linker lengths.

We report a significant improvement on RNA ligasemediated ligation (henceforth "Rligation") achieved in part through the incorporation of the $5^{\prime}$-silyl-2'-acetoxy ethyl orthoester $\left(2^{\prime}-\mathrm{ACE}\right)$ protecting group used in oligoribonucleotide synthesis (Hartsel et al. 2004), as well as through optimized linker lengths and chemically incorporated $5^{\prime}$ phosphates on the donor strand. RNA oligos containing $2^{\prime}$-ACE protecting groups are far less susceptible to intramolecular circularization than are corresponding $2^{\prime} \mathrm{OH}$ RNAs. These reactions proceed rapidly, some within $5 \mathrm{~min}$ or less, and give high yields of product. Notably, the ligation yields are high enough that it is now possible to create RNAs through multiple ligation steps, allowing site-specific modification of large RNAs. As an initial example, we have used this technique to produce with high efficiency a 128-nt pre-messenger RNA from three chemically synthesized oligonucleotide precursors. We describe the important parameters necessary to apply this ligation method to virtually any RNA sequence, and anticipate that this will facilitate the synthesis of a wide array of RNA molecules for detailed physico-chemical characterization.

\section{RESULTS AND DISCUSSION}

\section{Designing an RNA ligase method}

Many structure-function studies examining RNA biology are facilitated by the ability to insert site-specific modifications. Unsatisfied with the methods currently available for generating long RNA molecules containing site-specific modifications, we sought to optimize conditions necessary for producing such reagents. As a common problem in RNA ligase-mediated reactions is the production of side products, we sought a protocol that limited the number of possible reactions at each step to the single, desired reaction. Ideally, each step of the reaction should involve an acceptor molecule with a $3^{\prime} \mathrm{OH}$ and no $5^{\prime}$ phosphate, and a donor molecule with a $5^{\prime}$ monophosphate and no accessible $3^{\prime} \mathrm{OH}$. In this way, circularization of either substrate molecule would be impossible, reducing the amount of side product at each step. By keeping the donor molecule in its 2' $\mathrm{ACE}$ form, its $3^{\prime} \mathrm{OH}$ is rendered largely inaccessible, minimizing its ability to circularize and, therefore, the number of equivalents required for complete conversion of the acceptor molecule. The product of this reaction, after removal of the remaining $2^{\prime} \mathrm{ACE}$ protecting groups, would be an RNA terminated at both its $5^{\prime}$ and $3^{\prime}$ ends with free hydroxyls, making it an ideal acceptor molecule for subsequent reactions. Because the donor molecule can be added in sufficient excess to drive all of the acceptor molecules into product, this design, when implemented in the $5^{\prime}$ to $3^{\prime}$ direction, can produce RNAs of considerable size with reasonable efficiency.

To ascertain the efficiency of this method, we tested four unrelated ligation junctions, including the MD1 test sequence, two different positions in the transcript of yeast ORF YOL047c (including junction I shown in Fig. 1B), and one in the yeast U6 snRNA. As shown in Figure 2, the RNA ligase-mediated reactions yielded significant amounts of ligated product in all four cases. These reactions were run with donor (labeled B) in excess, so $100 \%$ conversion of acceptor (A) to products would result in the disappearance of the A band, as in panels 1 and 3. Note that the reactions in panels 1 and 2 were treated with DNase I after ligation to remove the splint oligo, which runs at the same position as the acceptor in those reactions. Under optimal reaction conditions (see below and Materials and Methods), apparent reaction yields were typically in the range of $70 \%-80 \%$ conversion of acceptor molecule into product, with many reactions proceeding to $\geq 95 \%$ conversion. Importantly, many reactions were complete within $5 \mathrm{~min}$, the shortest time measured, which is considerably faster than other reported ligation methods. The reactions shown in Figure 2 


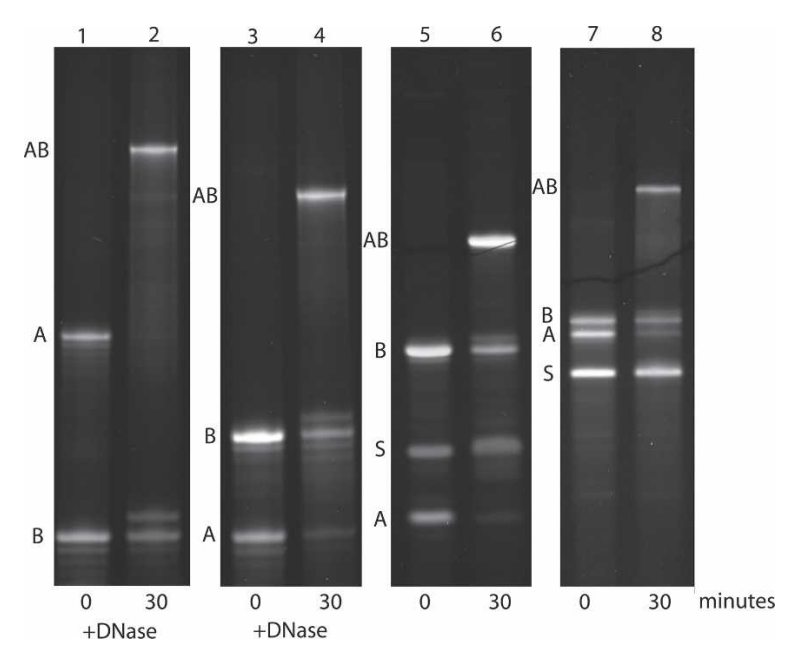

FIGURE 2. Rligation efficiency for four RNA junctions. Ligation reactions were performed as described in Materials and Methods and monitored by polyacrylamide gel electrophoresis (PAGE) followed by ethidium bromide staining. RNA used: (lanes 1,2) YOL047c junction I shown in Figure 1B; (lanes 3,4) YOL047c junction II; (lanes 5,6) MD1 RNA; (lanes 7,8) U6 snRNA. The corresponding splints are shown in Materials and Methods. The Rligation reactions in lanes 1-4 were treated with 1 unit of DNAse I for $15 \mathrm{~min}$ at $37^{\circ} \mathrm{C}$ following ligation in order to remove the DNA splint, which comigrates with the donor (oligo B). The locations of the acceptor oligos (A), the donor oligos $(B)$, and the ligated products $(A B)$ are indicated for each of the reactions.

were visualized by staining with ethidium bromide after complete deprotection of the reactions. Because $2^{\prime} \mathrm{ACE}$ containing oligonucleotides are poorly stained by ethidium bromide, the donor oligo is only visible as a weak, fuzzy band, unless it has been deprotected.

\section{Optimizing a ligation reaction}

To facilitate application of this method to a variety of RNA sequences, we sought to optimize the reaction parameters. In general, we found that the most important determinant of efficient ligation is the capacity to form a complex between the splint and the two substrates. We have examined ligation reactions using both nondenaturing and denaturing gels and found a high correlation between the amount of splint:substrate complex formed on the native gels and the amount of ligated product formed. Consistent with the recent observations using DNA ligase (Kurschat et al. 2005), formation of a splint:substrate complex appears to be required, if not sufficient, for ligation. With this in mind, ligation junctions were chosen at locations in which predicted secondary structures were most likely to be compatible with complex formation.

Previous work demonstrated that RNA ligase is inefficient when the ultimate or penultimate nucleotide of the acceptor molecule is a pyrimidine (Wittenberg and Uhlenbeck 1985). In the context of these splint:substrate complexes, we have examined multiple ligation reactions, including several containing 3 '-terminal pyrimidines in the acceptor oligo, and see no sequence specificity in either the donor or acceptor substrates (data not shown). We surmise that in the geometry achieved with a splint, the chemical steps of ligation are fast enough that the reaction becomes insensitive to the identity of the terminal nucleotides. It is possible that bulky nucleotide modifications (e.g., fluorescent dyes) at or near the ligation junction might interfere with the reaction, although we have not tested this directly. Modifications distal to the junction are unlikely to interfere, however, as they would be far from the catalytic site of the enzyme.

Once the ligation junction has been selected, the splint must be designed. The tRNA substrate of T4 RNA ligase has linker lengths of 5 and 2 nt on the acceptor and donor molecules, respectively. While the optimum lengths may vary for different sequences, our experience suggests that the 5:2 (acceptor:donor) combination is generally effective. Using the MD1 sequence, we independently varied the length of the linkers. For the donor molecule, efficiency drops off sharply for lengths other than 1 or 2 nt (data not shown). For the acceptor molecule, maximum activity was observed with eight single-stranded nucleotides, but high levels of ligation were also obtained with four to seven single-stranded nucleotides. Having chosen the single-stranded portions, it is also necessary to ensure stable association between the splint and the RNA substrates. The splints used in the ligations for Figure 2 were designed such that the melting temperature $\left(T_{M}\right)$ for each "half" of the splint was in the range of $40-45^{\circ} \mathrm{C}$. Our initial analysis suggested that a $T_{\mathrm{M}}<40^{\circ} \mathrm{C}$, especially between the splint and the donor, was particularly deleterious (data not shown).

When designing the splint, important consideration should be given to the possibility that the single-stranded linkers may inadvertently anneal to the splint. For example, it is important that the two linker nucleotides in the donor molecule (B) are not complementary to the last two nucleotides in the acceptor-binding region of the splint. Our original splint design incorporated the nucleotide purine, which is unable to participate in a stable base pair, between the donor- and acceptor-binding regions of the splint to prevent such base pairing. Splints containing nonstandard nucleotide spacers, such as purine, were highly active for ligation, but may slightly alter linkerlength optima. While we have not used a spacer in most of our splints, it may nevertheless be an effective strategy for improving efficiency in cases where cross-hybridization may occur.

We initially hypothesized that 2'ACE-containing RNA splints might be more effective than DNA splints, as the 2'ACE groups prevent tertiary structure formation (Scaringe 2001), and would therefore maintain an RNA splint in a more accessible conformation for base pairing to the 
substrates. In the cases where we have directly compared RNA and DNA splints, however, we see little or no difference (data not shown).

One potential disadvantage of Rligation compared to Dligation is the potential for heterogeneity at the ligation junction, resulting from the ability of the enzyme to accommodate a variety of lengths in the single-stranded region. This problem can be eliminated, however, by using chemically synthesized RNA. By their nature, the $3^{\prime}$ ends of synthetic oligos have a negligible amount of heterogeneity, ensuring the quality of the acceptor side of the junction. In contrast, the $5^{\prime}$ ends of synthetic oligos can be heterogeneous, resulting from truncated products of chemical synthesis. However, if the $5^{\prime}$ phosphate on the donor molecule is added during chemical synthesis of the molecule (as opposed to adding it post-synthetically using T4 polynucleotide kinase), one ensures that only full-length donor molecules will be ligated, since truncated products are chemically capped to prevent extension, and therefore will not contain the $5^{\prime}$ phosphate required for ligation. When using enzymatically produced RNAs, it will be important to ensure the purity of both the $3^{\prime}$ and the $5^{\prime}$ ends of the molecule (Pleiss et al. 1998) to guarantee junction fidelity as well as to maintain high yields. In such cases, sequencing the junction may be desirable.

Nontemplated ligation reactions can be detrimental to efficient ligation. Because donor molecules have the capacity to either circularize and/or concatamerize, we compared donor molecules in either the $2^{\prime} \mathrm{ACE}$ or $2^{\prime} \mathrm{OH}$ form. In initial experiments with the MD1 substrates, the ligation efficiency increased from $60 \%$ to $80 \%$ in the presence of the 2 'ACE groups while reducing the number of visible side products (data not shown). This finding is consistent with our observation that acceptor molecules are inactive when in the $2^{\prime} \mathrm{ACE}$ form, presumably because of steric inaccessibility of the $3^{\prime} \mathrm{OH}$. While we have not seen this magnitude of change in all of the reactions we have tested, donor molecules in the 2 'ACE form can generally be accommodated in the ligation scheme. We expect other $2^{\prime}$ protecting groups that render the $3^{\prime} \mathrm{OH}$ inaccessible to be equally effective in preventing side reactions. Other simple steps to avoid unwanted products include using splint oligos with a $3^{\prime}$ dideoxy nucleotide and terminating the $3^{\prime}$ terminal RNA oligo with a $2^{\prime}, 3^{\prime}$-dideoxy nucleotide.

\section{Proof of principle: Synthetic splicing reporter}

To establish the utility of the Rligation strategy in a biochemical context, we designed a pre-mRNA transcript made entirely of synthetic oligonucleotides (Fig. 3). We used the yeast intron database (Spingola et al. 1999) to identify intron-containing genes with introns shorter than $80 \mathrm{nt}$ so that our transcript could be made from three RNA oligos. We also looked for genes expressed at high levels in yeast, reasoning that these would be readily spliced, at least
A

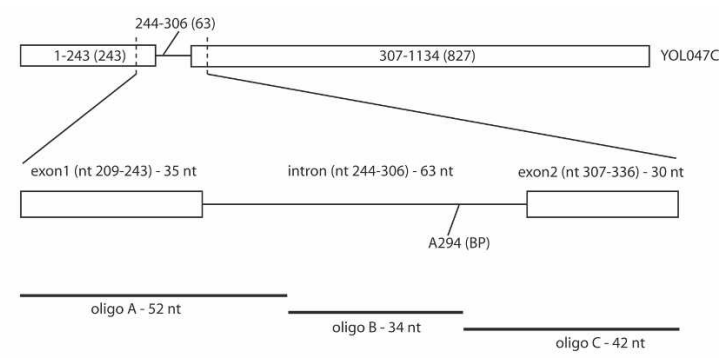

B

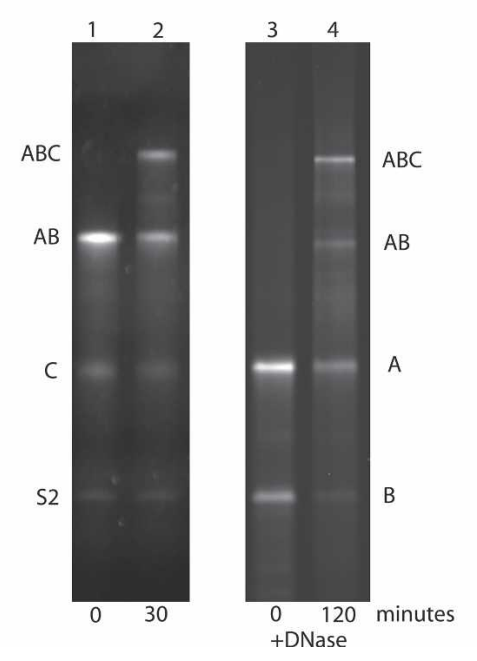

FIGURE 3. Ligation of 128-nt, synthetic pre-mRNA. (A) Strategy for making synthetic YOL047c transcript. The YOL047c gene contains a single 63-nt intron near the $5^{\prime}$ end of the gene. Dashed lines indicate boundaries of the splicing reporter. The 128-nt splicing reporter contains $35 \mathrm{nt}$ of exon 1, the entire intron (branch point nucleotide, $\mathrm{BP}$ ), and $30 \mathrm{nt}$ of exon 2. Synthetic oligoribonucleotides (labeled A, B, C) for generating the splicing reporter contain, respectively, the $5^{\prime}$ splice junction, the branch point, and the $3^{\prime}$ splice junction. (B) Twostep ligation of transcript (lanes 1,2). Gel-purified $\mathrm{AB}$ product (lane 1, "AB") was ligated with oligo C and splint S2, yielding full-length ABC product (lane 2, "ABC"). One-step ligation of transcript (lanes 3,4). All three RNA oligonucleotides and both splints were incubated in a single reaction. Reactions were treated with DNAse I after ligation, so no splints are visible.

in vivo. Using these criteria, we selected yeast open reading frame (ORF) YOL047c, an ORF of unknown function (Fig. $3 \mathrm{~A})$. We chose a region containing the entire intron (63 nt), plus $35 \mathrm{nt}$ of exon1 and $30 \mathrm{nt}$ of exon2, as our minimal splicing substrate, which we had synthesized in three fragments (oligos A, B, and C; Fig. 3A).

We attempted to generate our full-length product in two different ways. Our initial attempt utilized a stepwise approach where we first generated the $\mathrm{AB}$ ligation product (YOL047c ligation I), using splint S1 in a large-scale reaction under conditions similar to those shown in Figure 2. The second ligation step ( $A B$ ligated to $C$ ) was then carried out using gel-purified, deprotected $\mathrm{AB}$ (Fig. 3B, lanes 1 and $2)$. For comparison, we attempted to synthesize the $A B C$ product in a single-step, three-way ligation reaction simultaneously using all three oligos and both splints. For this 
reaction, piece $\mathrm{B}$ was deprotected prior to the ligation, as it is required to act both as a donor and an acceptor. As seen in Figure 3B, lanes 3 and 4, the single-step reaction was as efficient as using gel-purified AB. Presumably, the efficiency of the three-way ligation suggests that piece $B$ is not highly susceptible to deleterious side reactions, perhaps due to sequestration by the splints. While this may not be true for all three-way ligations, we have had success ligating several different sequences in this way (data not shown).

Our experience with this method suggests that this technique will prove useful for the ligation of RNA molecules of virtually any sequence. Indeed, the high efficiency of this technique, demonstrated by our synthesis of a 128nt RNA from three synthetic oligoribonucleotides, opens the possibility of modifying multiple positions in still longer RNA sequences. While we have exclusively used oligonucleotides in our experiments, we expect that in vitro transcribed RNA could be accommodated in our reaction scheme, as long as care is taken to ensure the homogeneity of the ligation junctions. When combined with the variety of modified nucleotides that can be incorporated during chemical synthesis, one can imagine creating site-specifically modified RNAs for use in a variety of structurefunction studies. We presume that the modifications would be less likely to interfere with ligation if the modification sites are not in the linker regions, although we have not tested this directly. We anticipate that an important application of this method will be for the incorporation of multiple fluorescent dyes into a single RNA molecule for use in fluorescence resonance energy transfer (FRET) experiments.

\section{MATERIALS AND METHODS}

\section{RNA oligo design}

Oligonucleotides were synthesized by Dharmacon using the 2'-ACE method on modified Applied Biosystems 380B synthesizers, using standard amidites. Potential RNA sequences were analyzed using m-fold (http://www.bioinfo.rpi.edu/applications/ mfold/old/rna/form1.cgi; Zucker 2003; Mathews et al. 1999) to determine the extent of secondary structure. Ligation sites were chosen either in regions with little or no intramolecular base pairing or at naturally occurring exon/intron junctions, with the constraint that oligos should not be significantly longer than 50 $\mathrm{nt}$. The longest oligo synthesized was $63 \mathrm{nt}$, but most were between 30 and $50 \mathrm{nt}$. Candidate junctions were analyzed for possible base pairing between the linker and the splint. The $3^{\prime}$ ends of the acceptor (A) oligos were in general designed to terminate in two purine bases, as it was originally thought that this provided a better substrate for T4 RNA ligase. The donor (B) oligos were synthesized with a $5^{\prime}$-terminal phosphate.

\section{DNA splint oligo design}

DNA oligos were purchased from Operon. DNA splints for the Rligation were designed to have $\sim 18$ nt of homology with the
RNA oligos on both the acceptor and donor halves, with melting temperatures for each half approximately equal to each other and $\geq 40^{\circ} \mathrm{C}$. $T_{\mathrm{M}}$ 's were calculated using nearest-neighbor thermodynamic parameters as implemented in IDT's OligoAnalyzer web tool (http://scitools.idtdna.com/scitools/Applications/OligoAnalyzer/ default.aspx; Xia et al. 1998).

\section{Rligation}

For all two-part ligations, the acceptor oligo (A) was deprotected and the donor oligo (B) was phosphorylated and 2'-ACE protected. The DNA splint oligo was mixed with the A and B RNA oligos at a 1:1.5:2 ratio of oligoA:splint:oligoB. Oligos were annealed $\left(65^{\circ} \mathrm{C}\right.$ for $3 \mathrm{~min}$ followed by $25^{\circ} \mathrm{C}$ for $5 \mathrm{~min}$ ) in the presence of $1 \times$ T4 RNA ligase buffer (NEB: $50 \mathrm{mM}$ Tris- $\mathrm{HCl}$ at $\mathrm{pH} 7.8,10 \mathrm{mM} \mathrm{MgCl} 2,10 \mathrm{mM}$ DTT, $1 \mathrm{mM}$ ATP). Annealing in the absence of ligase buffer results in at least partial deprotection of the B oligo, probably due to the acidity of our water, increasing the risk of circularization. Final oligo concentrations were between 2 and $4 \mu \mathrm{M}$. T4 RNA ligase (NEB) was added at a final concentration of $1 \mathrm{U} / \mu \mathrm{L}$ and the reactions were incubated at $37^{\circ} \mathrm{C}$, removing aliquots $(5$ or $10 \mu \mathrm{L})$ at the indicated times. Many reactions were complete in as little as $5 \mathrm{~min}$.

Samples in which the splint and one of the RNA oligos were too close in size to resolve on a gel were then treated with $1 \mathrm{U}$ DNAse I (Ambion) for $15 \mathrm{~min}$ at $37^{\circ} \mathrm{C}$, as indicated in the figure legends. All ligation reactions were stopped and deprotected by the addition of $100 \mu \mathrm{L} 2^{\prime}$-ACE deprotection buffer (100 mM acetate adjusted to $\mathrm{pH} 3.8$ with TEMED; Dharmacon), heated at $65^{\circ} \mathrm{C}$ for $30 \mathrm{~min}$, evaporated in a speed-vac, and resuspended in urea sample buffer. Ligation products were analyzed on 12\% TBE polyacrylamide/7 M urea gels and stained in $\mathrm{EtBr}(0.5 \mathrm{mg} / \mathrm{mL})$ after running.

The three-part ligation performed in two steps was done in the same way as the two-part ligation, with the first step scaled up to generate enough product to gel purify. The final A and B oligo concentrations were 50 and $100 \mu \mathrm{M}$, respectively, in a $20-\mu \mathrm{L}$ reaction, with $75 \mu \mathrm{M}$ splint. The ligation was stopped after $30 \mathrm{~min}$ by the addition of an equal volume of formamide, denatured at $65^{\circ} \mathrm{C}$ for $5 \mathrm{~min}$, and run on a $12 \%$ denaturing gel. The ligation products were isolated by UV shadowing and eluted from the gel. The purified $\mathrm{AB}$ product was deprotected as above to serve as the acceptor in the second step. The second step of the threepart ligation was then performed with the $\mathrm{AB}$ acceptor, $\mathrm{C}$ donor, and DNA splint 2 using equimolar amounts of the three components under the same conditions as for the original two-part ligation.

For the single-step three-part ligation the B oligo, as well as the A oligo, was deprotected, as it must serve as both a donor and an acceptor in this reaction. All five oligos were annealed to each other as above. Several different ratios of oligos were tested in an attempt to find optimal conditions. For the reaction shown in Figure 3B, the ratio of $\mathrm{A}: \mathrm{B}: \mathrm{C}$ was $2: 1: 2$, with a $\mathrm{B}$ :splint ratio of $1: 1.5$.

\section{Oligo sequences}

\section{YOLO47C ligation I}

oligo A r020 5'-AGUCGUCGCACCAGAUCAUCUGGUAAUCC GUAUAAAGUAUGUUGAUGUUCCAA-3' 
oligo B r021 5'-PUAAGCAGAUCAUGUUUUUUAAGCCGUCA UACUAA-3'

DNA splint oSDR171 5' '-TAAAAAACATGATCTGCTACATCAA CATACTTTATA-3'

\section{YOLO47C ligation II}

oligo A r028 5'-UAAGCAGAUCAUGUUUUUUAAGCCGUCA UACUAA-3'

oligo B r022 5'-PCCGCCUUUGAAGGGAAUUAGCGAAUCCG UGGGAAACUCGGAU-3'

DNA splint oSDR172 5'-CTAATTCCCTTCAAAGGCATGACGG CTTAAAAAACA-3'

\author{
YOLO47C ligation III \\ oligo A r020 5'-AGUCGUCGCACCAGAUCAUCUGGUAAUCC \\ GUAUAAAGUAUGUUGAUGUUCCAA-3' \\ oligo B r021 5'-PUAAGCAGAUCAUGUUUUUUAAGCCGUC \\ AUACUAA-3' \\ oligo C r022 5'-PCCGCCUUUGAAGGGAAUUAGCGAAUCC \\ GUGGGAAACUCGGAU-3' \\ DNA splint 1 oSDR171 5' ${ }^{\prime}$-TAAAAAACATGATCTGCTACATCA \\ ACATACTTTATA-3' \\ DNA splint 2 oSDR172 $5^{\prime}$-CTAATTCCCTTCAAAGGCATGAC \\ GGCTTAAAAAACA-3'
}

MD1

oligo A r001 5'-UGACCCGUCGAGAGAG-3'

oligo B r004 5' -PGAGAGUAGCCCAUGCAAUGCGAGGA-3'

DNA splint oSDR183 5'-GCATGGGCTACTTCGACGGGTCA-3'

\section{U6 SnRNA}

oligo A r006 5'-AUUUGAAACAAUACAGAGAUGAUCAGCAG UUCCCCUGC-3'

oligo B r007 5'-PAUAAGGAUGAACCGUUUUACAAAGAGAU UUAUUUCGUUUU-3'

DNA splint oSDR210 5'-GTAAAACGGTTCATCCTTGGAACT GCTGATCATCT-3'

\section{ACKNOWLEDGMENTS}

We thank Alexey Wolfson and Anastasia Khvorova for insightful discussions and Olke Uhlenbeck and Kelly Aukema for helpful comments on the manuscript. Dharmacon RNA Technologies assisted this work through a collaborative research agreement. This work was supported in part by NSERC Discovery Grant 298521 to S.D.R.

Received March 22, 2006; accepted August 3, 2006.

\section{REFERENCES}

Amitsur, M., Levitz, R., and Kaufmann, G. 1987. Bacteriophage T4 anticodon nuclease, polynucleotide kinase and RNA ligase reprocess the host lysine tRNA. EMBO J. 6: 2499-2503.

Bain, J.D. and Switzer, C. 1992. Regioselective ligation of oligoribonucleotides using DNA splints. Nucleic Acids Res. 20: 4372.

Chavatte, L., Frolova, L., Kisselev, L., and Favre, A. 2001. The polypeptide chain release factor eRF1 specifically contacts the $\mathrm{s}(4) \mathrm{UGA}$ stop codon located in the A site of eukaryotic ribosomes. Eur. J. Biochem. 268: 2896-2904.

England, T.E., Bruce, A.G., and Uhlenbeck, O.C. 1980. Specific labeling of 3' termini of RNA with T4 RNA ligase. Methods Enzymol. 65: 65-74.

Golden, B.L., Gooding, A.R., Podell, E.R., and Cech, T.R. 1996. X-ray crystallography of large RNAs: Heavy-atom derivatives by RNA engineering. RNA 2: 1295-1305.

Gumport, R.I. and Uhlenbeck, O.C. 1981. T4 RNA ligase as a nucleic acid synthesis and modification reagent. Gene Amplif. Anal. 2: 313-345.

Hartsel, S.A., Kitchen, D.E., Scaringe, S.A., and Marshall, W.S. 2004. RNA oligonucleotide synthesis via $5^{\prime}$-silyl-2' -orthoester chemistry. Methods Mol. Biol. 288: 33-50.

Heilek, G.M., Marusak, R., Meares, C.F., and Noller, H.F. 1995. Directed hydroxyl radical probing of $16 \mathrm{~S}$ rRNA using Fe(II) tethered to ribosomal protein S4. Proc. Natl. Acad. Sci. 92: 1113-1116.

Kurschat, W.C., Muller, J., Wombacher, R., and Helm, M. 2005. Optimizing splinted ligation of highly structured small RNAs. RNA 11: 1909-1914.

Mathews, D.H., Sabina, J., Zuker, M., and Turner, D.H. 1999. Expanded sequence dependence of thermodynamic parameters improves prediction of RNA secondary structure. J. Mol. Biol. 288: 911-940.

McGregor, A., Rao, M.V., Duckworth, G., Stockley, P.G., and Connolly, B.A. 1996. Preparation of oligoribonucleotides containing 4-thiouridine using Fpmp chemistry. Photo-crosslinking to RNA binding proteins using $350 \mathrm{~nm}$ irradiation. Nucleic Acids Res. 24: 3173-3180.

Moore, M.J. and Query, C.C. 2000. Joining of RNAs by splinted ligation. Methods Enzymol. 317: 109-123.

Moore, M.J. and Sharp, P.A. 1992. Site-specific modification of premRNA: The 2'-hydroxyl groups at the splice sites. Science 256: 992-997.

Pleiss, J.A., Derrick, M.L., and Uhlenbeck, O.C. 1998. T7 RNA polymerase produces $5^{\prime}$ end heterogeneity during in vitro transcription from certain templates. RNA 4: 1313-1317.

Romaniuk, P.J. and Uhlenbeck, O.C. 1983. Joining of RNA molecules with RNA ligase. Methods Enzymol. 100: 52-59.

Scaringe, S.A. 2001. RNA oligonucleotide synthesis via 5'-silyl-2'orthoester chemistry. Methods 23: 206-217.

Spingola, M., Grate, L., Haussler, D., and Ares Jr., M. 1999. Genomewide bioinformatic and molecular analysis of introns in Saccharomyces cerevisiae. RNA 5: 221-234.

Teigelkamp, S., Newman, A.J., and Beggs, J.D. 1995. Extensive interactions of PRP8 protein with the $5^{\prime}$ and $3^{\prime}$ splice sites during splicing suggest a role in stabilization of exon alignment by U5 snRNA. EMBO J. 14: 2602-2612.

Wittenberg, W.L. and Uhlenbeck, O.C. 1985. Specific replacement of functional groups of uridine-33 in yeast phenylalanine transfer ribonucleic acid. Biochemistry 24: 2705-2712.

Wyatt, J.R., Sontheimer, E.J., and Steitz, J.A. 1992. Site-specific crosslinking of mammalian U5 snRNP to the $5^{\prime}$ splice site before the first step of pre-mRNA splicing. Genes \& Dev. 6: 2542-2553.

Xia, T., SantaLucia Jr., J., Burkard, M.E., Kierzek, R., Schroeder, S.J., Jiao, X., Cox, C., and Turner, D.H. 1998. Thermodynamic parameters for an expanded nearest-neighbor model for formation of RNA duplexes with Watson-Crick base pairs. Biochemistry 37: 14719-14735.

Zuker, M. 2003. Mfold web server for nucleic acid folding and hybridization prediction. Nucleic Acids Res. 31: 3406-3415. 

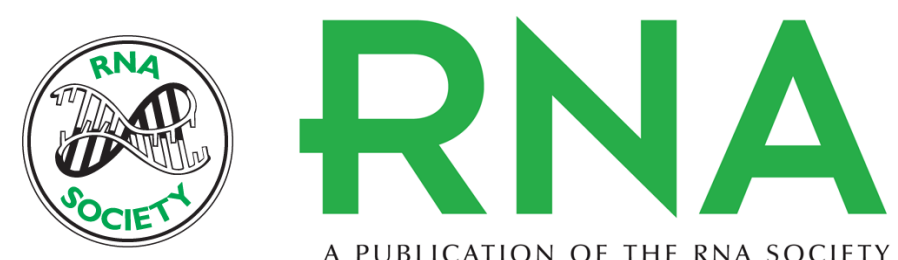

A PUBLICATION OF THE RNA SOCIETY

\section{An RNA ligase-mediated method for the efficient creation of large, synthetic RNAs}

Martha R. Stark, Jeffrey A. Pleiss, Michael Deras, et al.

RNA 2006 12: 2014-2019 originally published online September 18, 2006

Access the most recent version at doi:10.1261/rna.93506

References This article cites 22 articles, 7 of which can be accessed free at:

http://rnajournal.cshlp.org/content/12/11/2014.full.html\#ref-list-1

\section{License} Email Alerting $\begin{aligned} & \text { Receive free email alerts when new articles cite this article - sign up in the box at the } \\ & \text { Service }\end{aligned}$ top right corner of the article or click here.

To subscribe to $R N A$ go to:

http://rnajournal.cshlp.org/subscriptions 